\title{
Automated 2D airfoil optimization of intentionally choked blades
}

\author{
Eduard Braining \\ B\&B-AGEMA \\ braining@bub-agema.de \\ Aachen, NRW, Germany
}

\author{
Kristof Weidtmann \\ B\&B-AGEMA \\ weidtmann@bub-agema.de \\ Aachen, NRW, Germany
}

\author{
René Braun \\ B\&B-AGEMA \\ braun@bub-agema.de \\ Aachen, NRW, Germany
}

\author{
David Leonardo Hernandez \\ Rincon \\ RWTH Aachen University \\ david.hernandez@rwth- \\ aachen.de \\ Aachen, NRW, Germany
}

\author{
Dieter Bohn \\ RWTH Aachen University \\ dieter.bohn@rwth-aachen.de \\ Aachen, NRW, Germany
}

\section{ABSTRACT}

To keep up with the global efforts on the reduction of contaminant emissions and the development of more efficient energy conversion systems, gas turbine engineering strives constantly towards the improvement of each one of its components. The determinant role of turbomachinery blades on the thermodynamic behavior of the whole machine has captured for decades the attention of thermal and aerodynamics specialists that find in the optimization of airfoil profiles a promising and valuable way for the progress pointing to a cleaner energy industry.

The present work is motivated by the need of an application for the automated generation of efficient choked turbine cascades, since this property is usually seen as advantageous for design purposes and intentional choking is actually a common practice in the first turbine stage. Also, the fundamental physics of those flow characteristics have been widely studied by a large number of authors; there is still a lack of open literature related to the implementation of this principle for design purposes and the development of intentionally choked blades.

Therefore, a 3D model of a single stage close to choking conditions has been set up as reference model to prescribe the boundary conditions of the first vane and the conditions of the stage. Those conditions are used for a Blade-to-Blade (2D) optimization of the vane mid-section. The main concern of the 2D optimization is the realization of shock conditions at the throat section of the profile geometry, for the same boundary conditions. The philosophy of the optimization approach is related to an existing 2D design approach for subsonic flow, where the pressure distribution over the airfoil is characterized. A similar approach can be applied to choking blades, as the pressure distribution over an airfoil has a characteristic behavior. The comparison of the re- designed vane geometry shows a good agreement to the stage conditions of the reference model, while the shock at the throat section is more pronounced and the efficiency of the stage is kept on a high level.

\section{INTRODUCTION}

Within the design of modern turbines, early integration of automated design space exploration techniques in all stages of the turbine's design process is mandatory for achieving optimal results. Besides optimizing cycle parameters, the turbine itself is the most promising component to be optimized (Cofer et al., 1996). Numerous efforts have been taken in the past in order to increase the turbine`s efficiency by working on different topics. A big field for improving the turbine`s efficiency is the redesign and optimization of the airfoil`s shape by utilization of various optimization techniques as summarized by Bellucci et al. (2012) and the creation of airfoil shapes with welldistributed load for improved secondary flow behaviour (Weiß and Fottner, 1993).

In jet engines and gas turbines, the first stages have usually high expansion pressure ratios, which are leading to high flow Mach numbers in the cross-section and therefore the first nozzle is usually choked. The intentional choking of the first nozzle is usually seen as advantageous for design purposes and is actually a common practice in the first turbine stage (Baskharone, 2006), so the mass flow rate through the turbine is set constant and therefore, is independent from stage behavior of downstream rows. Nevertheless, the choke condition is ongoing with the occurrence of shocks, which have a high impact on the load distribution over the airfoil (Bohn et al., 1997, Figure 1) and the performance of the stage. Therefore, the common 
practice of the creation of airfoil shapes with well-distributed load, as suggested by Weiß et al. (1993) cannot be applied to intentionally choked nozzles. Still, choking rows are showing specific characteristics which follow a rule. The fundamental physics of those flow characteristics have been widely studied by a large number of authors. However, there is still a lack of open literature related to the implementation of this principle for the automated design purposes and the development of intentionally choked blades.

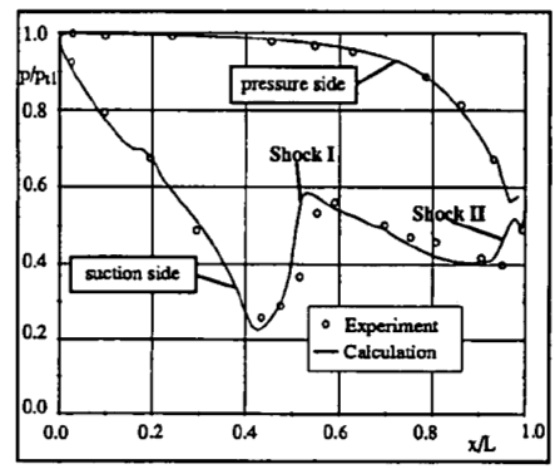

Figure 1: Example of the normalized static pressure distribution of a typical transonic turbine vane (Bohn, 1997)

For the common practice of automated airfoil optimization, it is only reasonable to extend existing approaches and adopt the characteristics of those flow patterns. Well-defined parameters, which described those flow patterns, are crucial for the analysis of an optimizationtask and for the success of an optimization process. The content of this paper is based and related to previous studies of the development of an automated design process which features blade to blade (B2B) optimizations performed by the authors and presented by Weidtmann et al. (2017). The extended approach of a 2D B2B optimization (Braining et al., 2018) is used as basic approach for the implementation of criteria to adopt an automated optimization approach for intentionally choked blades. Therefore, the characteristics of a choked blade are analysed and an objective function is adopted. A 3D stage model is utilized for providing the boundary conditions and for the validation of the $2 \mathrm{D}$ B2B optimization of the first vane, which targets the realization of shock conditions at the throat section of the profile geometry.

\section{CHARACTERISTICS OF CHOKE \& SHOCK}

Considering a convergent-divergent nozzle of length 1 , provided with a valve to control the exit static pressure from the stagnation value $\mathrm{p} 2=\mathrm{pt} 1$ at the starting closed position 0 . As the valve starts to open, the mass flow increases and the static pressure drops (see Figure 2). This behavior continues until the Mach number at the smallest cross section is equal to the unity and the pressure in this position is critical. At this point (2), the mass flow reaches its maximum and cannot be modified by additional exit pressure reductions. This state is called choke condition and is of essential importance in transonic flows (Korpela, 2011).

A further reduction of the outlet pressure will lead to a sudden change of flow variables through the formation of a normal shock wave. Furthermore, the position of the shock wave is depending on the pressure ratio and with higher pressure ratios, the shock wave will migrate downstream towards the outlet plane. If the flow is under expanded or over expanded, other oblique shock waves or expansion fans will find place behind the nozzle and the back pressure would be matched (Denton et al., 1989).
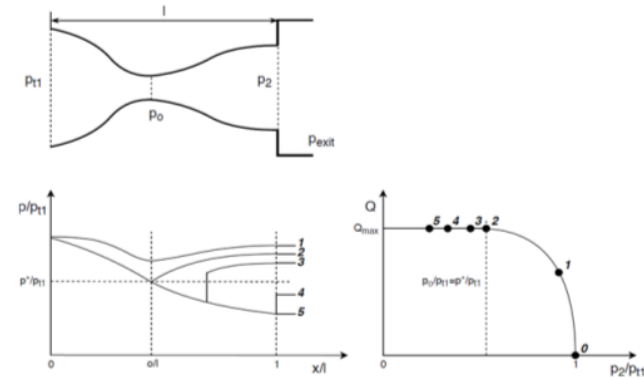

Figure 2: Scheme of a nozzle, pressure development and flow function at different outlet pressures

The first sign of choking is the appearance of supercritical regions over the suction side (SS) near the throat, which are identified by a negative peak in the pressure distribution (similar to Figure 1). The pressure jump linked to this peak is usually quantified in the transonic turbine literature through a parameter called diffusion factor (DFAC), which relates the difference between the maximal Mach number and its contiguous minimum immediately after passing the shock. Analogous to the definition based on the Mach number, DFAC can be written as a function of the shock pressure ratio:

$$
D F A C=\frac{\left(p_{s s, \text { max }}\right)_{x>x, S S, \text { min }}-p_{s s, \text { min }}}{p_{S S, \text { min }}}
$$

A high value of DFAC would indicate the presence of at least one shock wave over the suction surface, which is in fact, a mandatory requirement for the development of critical conditions in a cascade. Findings published by Corriveau and Sjolander (2003) suggest the existence of a relation between DFAC and the total cascade losses. Although the researchers do not formulate any correlation for it, they argue a direct influence of DFAC on the blade efficiency.



Figure 3: Loss breakdown of a typical transonic cascade at different Mach numbers (Mee, 1990) 
Mee et al. (1990) have shown through a set of measurements that the three components of loss in turbine cascades are strongly influenced by the Mach number and display a consistent behavior corresponding to the models that describe them. These models are well depicted in the thoroughly study on the entropy production and the physics of two dimensional cascades presented by Denton (1993). Neglecting the losses induced by heat transfer (regarding adiabatic conditions), the overall loss coefficient in any two dimensional cascade is the result of the sum of the blade boundary layer losses, the shock wave losses and the trailing edge losses (see Figure 3).

The boundary losses are decreasing with increasing outlet Mach number (higher pressure ratio $\mathrm{p} 2 / \mathrm{pt} 1$ ). The losses due to shock wave are significant at $0.95<\mathrm{M}_{2}<1.1$. At higher pressure ratios, the shock wave turns oblique and become weaker. A further increase of the pressure ratio leads to a reduction of the shock losses.

While the outlet Mach number is lower than one, the boundary losses are dominant. At outlet Mach numbers above $\mathrm{M}_{2}>1$, the mixing losses are the dominant factor of the total losses. According to turbulence theory, the erratic movement of the fluid particles in vortical structures procures and accelerates mixing processes, for this reason, the entropy creation related to shear outside the BL is generalized under the concept of mixing losses and coincides with the notation of Mee et. al (1990). Since a great part of the total mixing in a cascade occurs behind the trailing edge (TE) and especially within the wake, Denton has proposed a general approach for the computation of the viscous losses outside of the boundary layer and names them trailing edge losses. He had demonstrated that the total pressure loss coefficient in this region can be understood as the sum of three concurrent effects: the loss related to the decay up to the low pressure on the dead water zone behind the trailing edge shock (known as base pressure pb), the mixing between the main stream in the central part of the passage and the low velocity stream coming from the boundary layer and the change in blockage in the cascade passing the blade row (Denton, 19993).

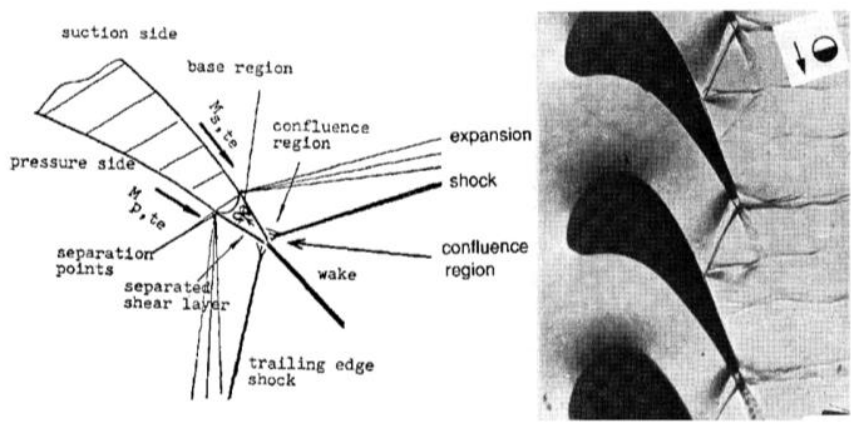

Figure 4: Schematic representation of the trailing edge shock system in supersonic flow (Denton, 1989)

Of importance is the formation of the trailing edge shock which is illustrated in Figure 4. The formation of this structure is of high relevance, given the fact that is almost unavoidable and has great impact in the flow behaviour.

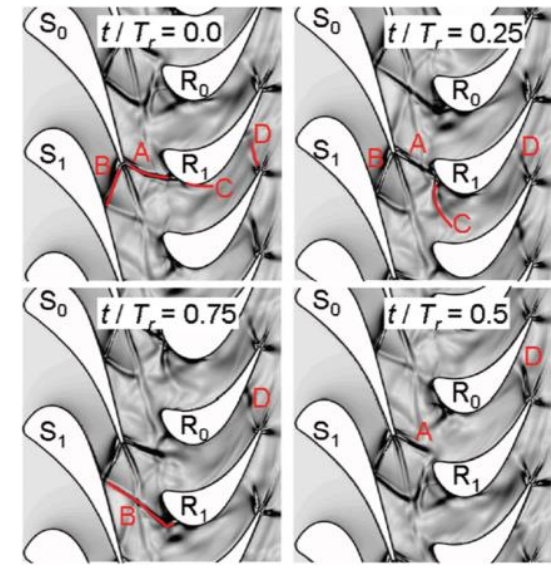

Figure 5: Findings of the unsteady RANS simulations on the stator-rotor blade interaction in a transonic turbine (Paniagua, 2008)

The shock system is not only by itself related with high amounts of entropy production. Summed to the viscous losses at the separated shear layers, the non-frictional entropy gained by passing the compression shocks and the effects on the main passage and the boundary layer of the adjacent blade, it has been proved that the TE shock branches do influence the behaviour of the downstream rows. Paniagua et.al (2008) has exposed that the periodic impingement of the TESS shock and the reflection of TEPS shock in a NGV row affects the flow of the next rotor annulus, causing further unwanted shock reflections (see Figure 5) and disturbances inside the blade passage. Bohn et.al (2005) has also evidenced adverse vibration stimulation due to fluctuations on the unsteady pressure profiles promoted by the statorrotor interactions. Other detrimental effects on performance have been evidenced by other authors (e.g. Giles (1990)) what suggests a beneficial effect through the intentional reduction or suppression of the propagation of TESS shock. Trying to minimize this influencing factor is an actual research topic.

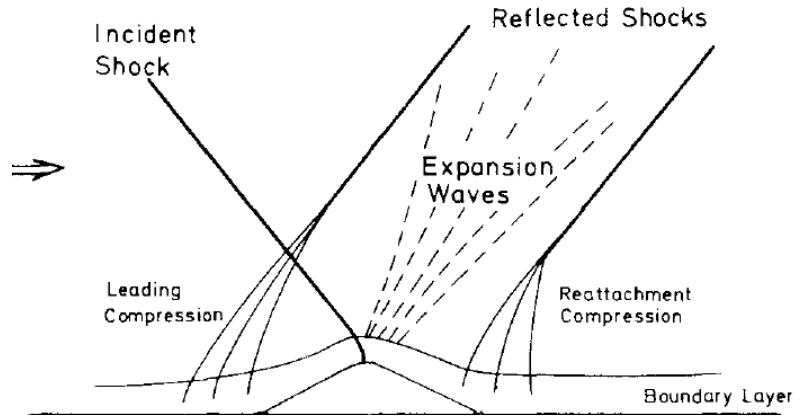

\section{Figure 6: Schematic representation of the SWBLI when a shock impinges a separation Bubble} (Graham, 1979)

In any supersonic flow over a wall, the presence of the boundary layer implies the existence of a subsonic region immediately near to the surface. The formation of a shock wave in any viscous flow, thus presuppose a reciprocal influence of transonic and dissipative phenomena arising from the interaction between the main stream and the flow inside the BL. This complex effect has been widely studied 
and is known as shock-wave/boundary-layer interaction (SWBLI). The schematic presentation of the SWBLI for the exposed situation of an impinging shock over the separation bubble is shown in Figure 6. Studies by Pearcey and Alber have shown, that for individual airfoils, separation could only be measured when the Mach number before the shock has a value above 1.3 and the kink that establishes the pressure downstream of the shock coincides with the critical condition.

\section{BASIC APPROACH}

For the common practice of automated airfoil optimization, it is only reasonable to extend existing approaches and adopt the characteristics of intentionally choked airfoil blades. The most vital part of automated airfoil optimization is the definition of criteria, which either described the shape of an airfoil section or the pressure distribution over the airfoil. Those approaches are then coupled in $2 \mathrm{D}$ or $3 \mathrm{D}$ simulations to obtain airfoil sections to create 3D CAD models and to provide the desired flow distribution to the prescribed boundary conditions.

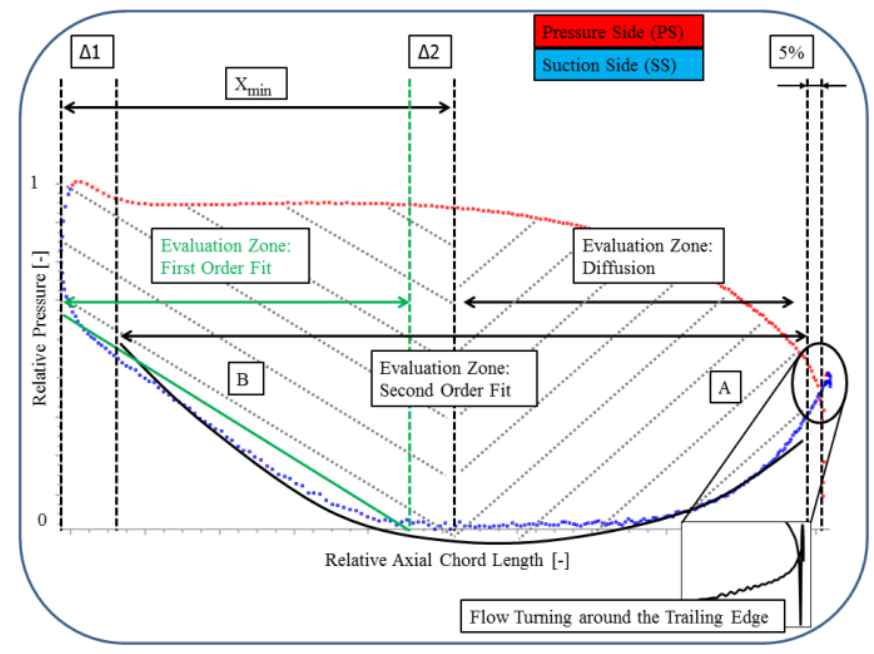

\section{Figure 7: Characterization of the pressure distribution for automated design approach of subsonic flow}

One possibility to realize such a task is the description of the pressure distribution over an airfoil by the definition of curve fits, as it has been discussed by Goel (2009). Based on this approach, the calculated pressure load is measured on the suction/pressure side and then, the deviation to the approximation of the pressure load by a curve fit is evaluated, analyzed and rated. This procedure allows one, to prescribe properties of a desired shape of the pressure distribution over an airfoil section; incidence, smooth acceleration in the front part, location of pressure minimum and diffusion.

In previous studies, performed by the authors, the approach has been valid as robust and effective for subsonic flow and has been embedded in the own design process. Therefore, the basic thoughts made by Goel (2009) have been adjusted to the own requirements and have been implemented in an objective-function. This was necessary, to consider the different criteria in a single objective for the optimization process. Thus, an opposing behavior between some criteria can also be taken into account. Furthermore, the calculated deviations between the approximated and calculated pressure distribution are weighted against each other in means of pressure load, velocities or the relative location of the pressure minimum. An overview is given in Figure 7.

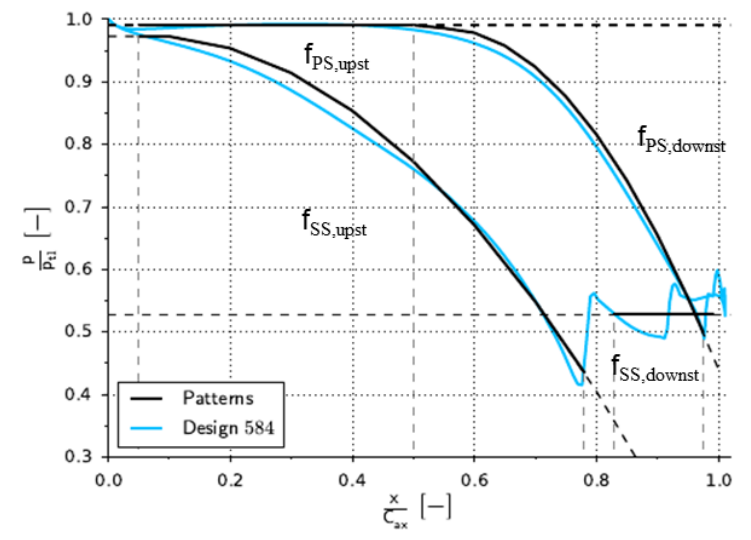

Objective Function $=F=f_{s S_{,}, \text {ppst }}+f_{s S_{\text {,downst }}}+f_{P S, \text { upst }}+$ fPS, downst $_{\text {, }}$

$$
\begin{aligned}
\mathrm{f}_{\mathrm{i}, \mathrm{j}}=\mathrm{WF} \bullet & \mathrm{Y}_{\mathrm{i}, \mathrm{j}} \\
\mathrm{WF} & =\text { Deviation (mean square error) } \\
Y_{\mathrm{i}, \mathrm{j}} & =\text { Pressure Function }
\end{aligned}
$$

\section{Figure 8: Characterization of pressure distribution for transonic flow and scheme of the objective function $\mathrm{F}$}

For subsonic flow, the approach has been confirmed as suitable for an automated design process. In transonic passage flow, the approach has to be modified for the analysis and the description of well pronounced shock systems.

A typical shape of the pressure distribution of intentional choked airfoil is pictured in Figure 8. It is to expect, that on the suction side the flow is continuously accelerated until the formation of a shock wave (pressure jump). Investigations have shown, that for a first estimation a square function with low diffusion is a good approximation of the behavior upstream the shock wave $\left(\mathrm{WF}_{\mathrm{SS}, \mathrm{Upst}}\right)$. The same behavior has been observed on the pressure side, downstream the throat position $\left(\mathrm{WF}_{\text {PS,Downst }}\right)$. Upstream the throat position, a constant pressure level on the pressure side is reasonable to keep the friction loses on a low level, which is realized by a linear approximation $\left(\mathrm{WF}_{\mathrm{PS} \text {,Upst }}\right)$. Also on the suction side, downstream the shock wave, the pressure is assumed to be constant $\left(\mathrm{WF}_{\mathrm{SS} \text {,Downst, }}\right.$ linear approximation). It is to note, that an assumption of constant pressure in this region is the designers decision to simplify the realization of the automated process.

Each of those deviations is furthermore weighted by a pressure function $\left(\mathrm{Y}_{\mathrm{i}, \mathrm{j}}\right)$. The pressure functions are including pressure ratios to balance the deviations against each other; as incidence, base pressure, trailing edge pressure, total to static ratio, critical pressure ratio of the row, minimal pressure on the suction side and the diffusion factor DFAC. By doing so, the base pressure, the trailing edge pressure and 
DFAC are meant to be maximized, while the incidence and the minimum pressure on the suction side are meant to minimize. The total to static ratio is given by the boundary conditions and the critical pressure ratio is kept constant. Furthermore, the shock formation is expected at the throat section thus, mid-loaded profiles are preferred. The objective function $\mathrm{F}$ can be seen as a geometric approach to prescribe the properties of intentional choked airfoils. Thus, within the optimization the profile parameter are adjusted to have a small deviation between CFD and curve fits. It can be said, that the profile parameter are optimized to the pre-defined properties of the pressure distribution and not to a specific shape of the pressure distribution.

\section{SIMULATION MODEL AND SETUP}



Figure 9: 3D Simulation model and mesh resolution

A 3D stage model of a single stage close to choking conditions is used as reference case, for prescribing the boundary conditions for the optimization approach and validation of the 2D B2B optimization results. The model and the mesh resolution are shown in Figure 9. The grid convergence method (Roache et. al, 2008) has been utilized (GCI) for the investigation of mesh-independency. Three mesh densities (coarse, medium and fine) have been investigated and the results of the mesh-study are shown in Table 1 (3D, right). At the mixing plane between vane and blade, the deviation of the values from coarse to medium mesh (GCI21) are already on a low level. Also the comparison between medium and fine mesh is showing a low deviation (GCI32). Therefore the medium mesh has been used for the investigations, with 5.3 million volume cells.

Table 1: Tracked values of the mesh independence study

\begin{tabular}{|l|c|c|l|l|}
\hline & \multicolumn{2}{|c|}{$2 \mathrm{D}$} & \multicolumn{2}{c|}{$3 \mathrm{D}$} \\
\hline Value & $\mathrm{GCI}_{21}$ & $\mathrm{GCI}_{32}$ & $\mathrm{GCI}_{21}$ & \multicolumn{1}{c|}{$\mathrm{GCI}_{32}$} \\
\hline $\mathrm{T}_{\mathrm{t}, \mathrm{mxp}}$ & $0.09 \%$ & $0.11 \%$ & $0.03 \%$ & $0.06 \%$ \\
\hline $\mathrm{T}_{\operatorname{mxp}}$ & $0.26 \%$ & $0.22 \%$ & $0.13 \%$ & $0.5 \%$ \\
\hline $\mathrm{p}_{\mathrm{t}, \mathrm{mxp}}$ & $0.07 \%$ & $0.18 \%$ & $0.06 \%$ & $0.16 \%$ \\
\hline $\mathrm{p}_{\operatorname{mxp}}$ & - & - & $0.01 \%$ & $0.16 \%$ \\
\hline
\end{tabular}

An overview of the stage performance of the reference case is shown in Table 2 for the medium mesh. It is to note that the geometry of the stage has been simplified. The first vane does not consider film cooling, only sealing flow and cooling flow at the cut back are modelled. Furthermore, the airfoil blade of the first vane is determined by one section and the shape is constant along the span. This $3 \mathrm{D}$ calculation of reference geometry is the base for the rating of the optimization process.

Table 2: Global stage parameter of the reference case

\begin{tabular}{|l|l|}
\hline Total temperature ratio $(\Theta)$ & 0.797 \\
\hline Total pressure ratio $(\Pi)$ & 0.408 \\
\hline Stage Loading $(\Psi)$ & -1.56 \\
\hline Reaction $(\rho)$ & 0.35 \\
\hline
\end{tabular}

In the optimization process the first vane is meant to be optimized based on one profile (50\% span). The focus of the optimization process is the realization of intentional choked profiles and improving the stage performance is secondary. For the automated approach, the mid-section is optimized in a 2D blade to blade simulation, which is coupled with the optimization algorithm SHERPA (Optimizer-HEEDs). An overview of the optimization chain is given in Figure 10. The optimizer HEEDs includes the calculation model. Furthermore, the constraints and the profile parameter are defined in HEEDs. The optimizer is responsible for the variation of profile parameter, execution of the calculation model and the rating of the results based on the constraints. The profile creator itself is coupled within the CFD simulation model. Furthermore, the optimizer is capable of executing post process scripts within the calculation model, which are necessary for the exchange of profile geometry and the evaluation of the objective function. Therefore, the results of the CFD calculation are extracted and provided to HEEDs.

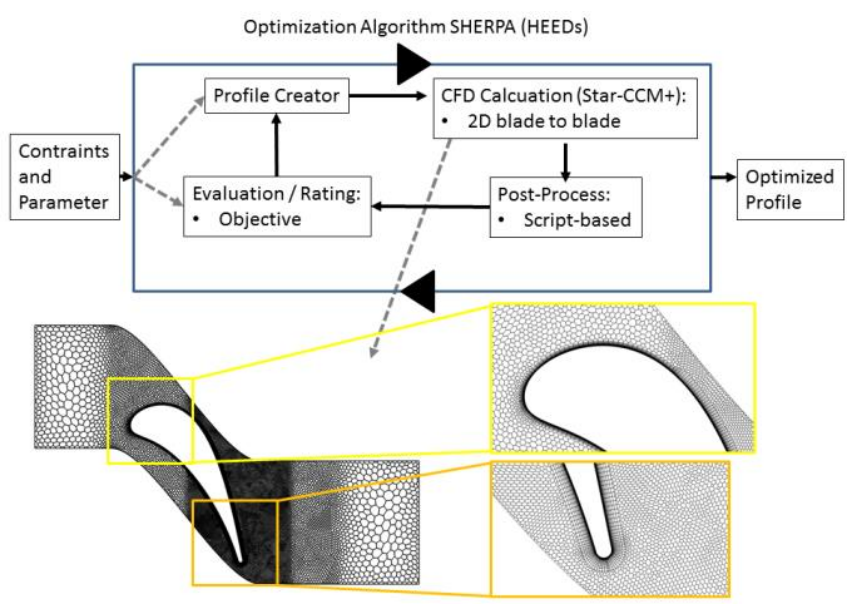

\section{Figure 10: Scheme of the automated approach and the $2 \mathrm{D}$ calculation model}

The optimization process is determined by the parameter of the profile creator and constraints based on the 3D reference case (case R). Therefore, the reference geometry is calculated in $3 \mathrm{D}$ and the boundary conditions for the $2 \mathrm{D}$ optimization (Table 3) are extracted at 50\% span (total 
pressure/temperature, static pressure, flow turning etc.). For the profile parameter a wide range has been considered. Thus, new profile geometry should be found by the optimization process which is fitting the boundary conditions and the constraints. The definition of the objective function and its validation is the main work of this study. The objective of the optimization process is the realization of shock-formation at the throat section of the blade passage. The 2D calculation domain of the optimization is shown in Figure 10. The mesh resolution of the calculation model has been increased in the area of interest, in the same way as for $3 \mathrm{D}$ reference case.

Table 3: Boundary Conditions for the 2D Calculation

\begin{tabular}{|c|c|}
\hline $\mathrm{p}_{2} / \mathrm{p}_{\mathrm{t} 1}[-]$ & 0.568 \\
\hline $\mathrm{T}_{2} / \mathrm{T}_{\mathrm{t} 1}[-]$ & 0.883 \\
\hline$\beta_{1}-\beta_{2}\left[{ }^{\circ}\right]$ & 73.4 \\
\hline
\end{tabular}

\section{RESULTS AND DISCUSSION}

The objective function $F$ (Figure 8) has been implemented in the automated 2D blade to blade optimization approach and several studies have been carried out. In the first investigation, the function is considered as the only objective. In the second and third investigation, the objective of the optimization has been defined as the weighted sum of function $F$ and the isentropic efficiency, while the weighting factor of isentropic efficiency has been changed. The results of those investigations have been verified in $3 \mathrm{D}$ calculations. Furthermore, the optimization approach has been carried out at higher total to static pressure ratios, to verify the capabilities of the approach. The investigations can be summarized as followed:

- A: Objective: Function F

- B: Objective: Function $\mathrm{G}$ with $\mathrm{X}_{\mathrm{i}}=1$

- C: Objective: Function $\mathrm{G}^{*}$ with $\mathrm{X}_{\mathrm{i}}=0.125$

- D: Based on $\mathrm{C}$, with increased pressure ratio

The function $G$ is defined in Equation 2. The "Ref" values of efficiency and function $\mathrm{F}$ are calculated for the first design of the optimization study, which is the reference geometry. The weighting factor $\mathrm{Xj}$ is constant $(=1)$ for all studies, while $\mathrm{Xi}$ is set to 1 in study $\mathrm{B}$ and to 0.125 in study C. Thus, the contribution of efficiency to the objective value of the optimization study is increased from A to B and from $\mathrm{B}$ to $\mathrm{C}$.

$$
G=\frac{\eta_{s}}{\eta_{s, \operatorname{Re} f}} * X_{i}+\frac{F}{F_{\operatorname{Re} f}} * X_{j}
$$

For study A to C 1500 designs have been explored and about $20 \%$ of the calculated designs have been declared as feasible designs. The best designs of each study, in terms of geometry, pressure distribution and performance are pictured in Figure 11. On the left, the profile shapes and on the right the pressure distributions are compared between reference case and the explored profiles.

As it can be observed, the pressure distribution of the reference case (black line) has its pressure minimum near the
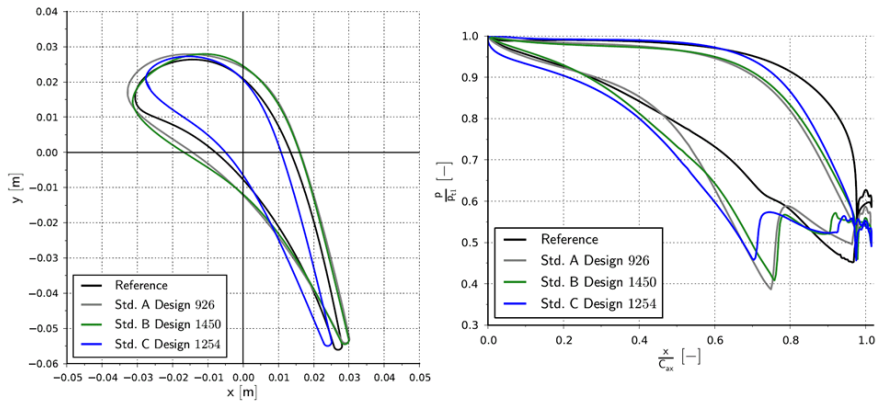

\section{Figure 11: Comparison of the geometry (left) and pressure distribution (right)}

trailing edge, which is followed by pressure increase due to the formation of a shock wave. It can be stated, that for the reference boundary conditions the choke conditions are not realized with the reference geometry. By applying the function $\mathrm{F}$ as objective for the optimization approach, the pressure distribution shows the typical behaviour at the throat sections (grey line). It is to expect, that choking is realized for this case. Furthermore, a smooth behaviour of the pressure distribution can be observed on the pressure side. On the suction side, moderate acceleration of the flow can be observed around the leading edge. Further downstream, the velocities are strongly increasing up to the throat section and the formation of a shock wave is clearly seen at the throat. Downstream the throat the flow is slightly re-accelerated and a second local shock can be observed, which is close to the trailing edge. In comparison to the reference case, the pressure in this region is increased. Furthermore, the profile is thicker in comparison to the reference case (Figure 11, left). Overall the performance is lower and the pressure losses of design 926 are increased, compared to reference geometry. Also the desired flow patterns are realized, a better performance of the profile is desirable for an automated design process.

In study $\mathrm{B}$, the objective is the weighted sum of function $\mathrm{F}$ and isentropic efficiency. The weighting factor $\mathrm{X}$ is 1 for both values. The pressure distribution (green line) is nearly the same as for study A. On the suction side, the flow acceleration is smoother in comparison to study A. Furthermore, the diffusion factor DFAC is reduced as the pressure jump at the throat section is smaller. Thus, the reincrease of pressure after the shock wave is lower. The second local shock on the suction is located further upstream the trailing edge and occurs at higher pressure level. Thus, the local shock is weaker in comparison to study A and the pressure near the trailing is on a lower level. A difference of the geometry can only be observed near the leading edge. By considering the objective $\mathrm{G}$, the performance of the profile have been slightly improved, but the impact of the efficiency is low. Due to the definition of $G$, the change of function $F$ is greater than the change of isentropic efficiency and therefore, the contribution of function $F$ to the value $G$ is greater. Also the contribution of the loss parameter to the objective value is low, an impact on the results can be observed.

To increase the contribution of the performance to the objective value $G$, the weighting factor $X_{i}$ has been set to 


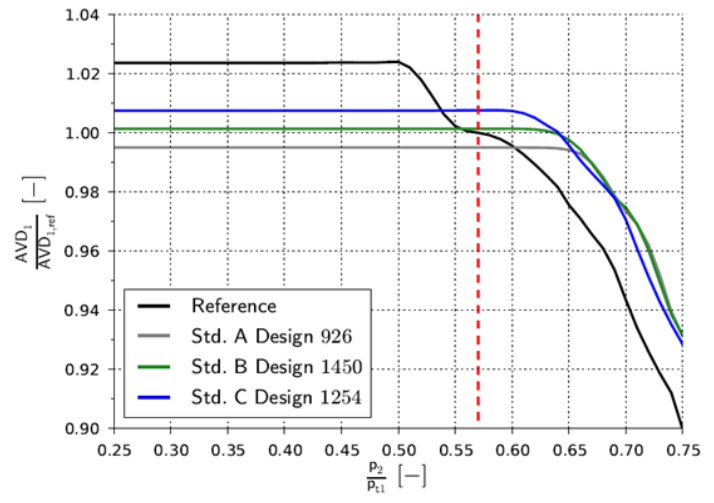

Figure 12: Comparison of the selected profiles at different pressure ratios

0.125 (Study C, blue line). It can be observed, that the shock wave is located further upstream and the diffusion factor DFAC is smaller in comparison to study A and study B. The flow behaviour downstream the shock wave is similar to the profile of study B. As it has been stated by Corriveau and Sjolander (2003) the value of DFAC has a relation to the total losses in the blade passage. Furthermore, lower pressures on the suctions side close to the trailing edge do further reduce the losses. Overall, the performance of the profile can be improved by considering the isentropic efficiency and the function $\mathrm{F}$, since in the design process a good performance of a profile is as important, as the conditions which have to be realized. It is interesting to observe, that the profile itself is much thinner in comparison to study A, which would also indicate lower friction losses.

To verify the choke conditions, all profiles have been calculated at different pressure ratio (see Figure 12). The reference conditions are determined by the red dashed line. It can be seen, that the reference geometry is choking under lower static to total pressure ratios as they are present. The profiles obtained in study A and B are even choking under higher pressure ratios, which is actually not necessary and not desired. In terms of choking, the profile obtained from study $\mathrm{C}$ provides the best results and the performance is on a comparable level as the reference geometry. The approach with the objective $\mathrm{G}^{*}$ (study $\mathrm{C}$ ) seems to be suitable to provide choking conditions for reference boundary conditions in a 2D blade to blade calculation.

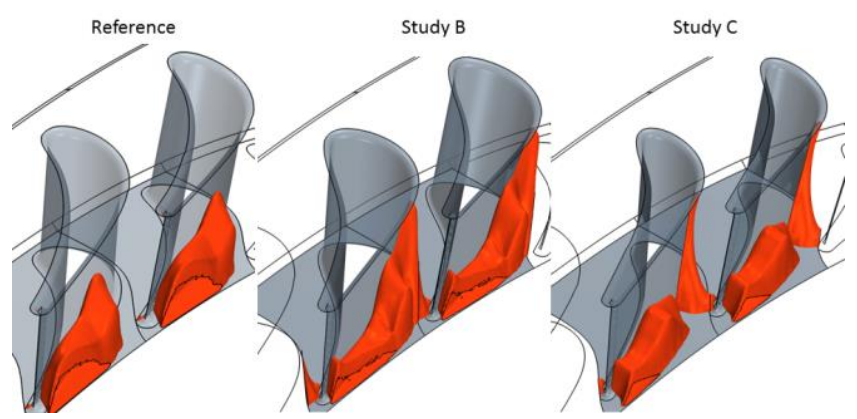

Figure 13: Comparison of reference, study B \& C in 3D calculation, iso-surface of sonic conditions is pictured

The profiles from study $\mathrm{B}$ and $\mathrm{C}$ have been further calculated in a 3D simulation. In Figure 13, an iso-surface of the sonic condition is presented in the 3D blade passage. From left to right, the reference geometry, the geometry from study $\mathrm{B}$ and the geometry from study $\mathrm{C}$ are pictured. It is to note, that the geometry of the stator-blades is constant in radial direction. The mid-section is extruded in both directions.

Again, it is evident that the reference geometry does not provide choke conditions in the blade passage. The region of high Mach numbers is located downstream the throat. Furthermore, sonic conditions are only present at lower spans (below 0.5). In contrast, the blade geometry from study B provides clear sonic conditions in the throat section over the whole span. The focus of function F seems to fit the task of applying the prescribed properties, also for simplified blade geometry. For the profile of study $\mathrm{C}$, the sonic region is also present at the throat section but is not fully developed over the cross section in radial direction. Nevertheless, the characteristics which have been prescribed in $2 \mathrm{D}$ are also present in the 3D simulation. The extension of the 2D approach to $3 \mathrm{D}$, for the individual design of the blade geometry may solve the issue of providing clear choke conditions over the whole span, while the loss generation can be held on a low level.

In Table 4, the global parameters of the stage are compared to each other between the different cases. Both cases, B and C, are in a good agreement to the reference geometry. In study $\mathrm{B}$, the mass flow and power output are slightly reduced. The geometry of study $\mathrm{C}$ provides a slightly increased mass flow rate and power output. Overall the deviation between reference and case $\mathrm{C}$ is smaller in comparison to case B. Furthermore, the efficiency is slightly increased compared to the reference case, which may also be a result of the increased mass flow rate. The deviation of the mass flow rate between case $\mathrm{B}$ and $\mathrm{C}$ is approximately $1 \%$ point; the deviation of the power out is more than $1 \% \mathrm{pt}$. Thus, the increased mass flow rate cannot be the only reason for the increased power out in case C. The deviation of efficiency in comparison to reference is moderate or minor. But, clear choke conditions are realized in both cases in comparison to the reference (see Figure 13), which are located at the throat position.

Table 4: Comparison of the global stage parameter

\begin{tabular}{|l|c|l|l|}
\hline Value & Reference & Study B & Study C \\
\hline$\Delta \dot{m}[\%]$ & - & -0.748 & 0.307 \\
\hline$\Delta \eta[\%]$ & - & -0.142 & 0.121 \\
\hline$\Delta \mathrm{P}[\%]$ & - & -0.857 & 0.642 \\
\hline$\psi[-]$ & -1.556 & -1.551 & -1.577 \\
\hline$\rho[-]$ & 0.35 & 0.34 & 0.356 \\
\hline$\Pi[-]$ & 0.408 & 0.407 & 0.4075 \\
\hline
\end{tabular}

An additional study has been carried out, where the profile is optimized to a lower static to total pressure ratio (0.5) in 2D simulation, with the objective $\mathrm{G}^{*}$. Furthermore, the profiles of study $\mathrm{C}$, study $\mathrm{D}$ and the reference geometry have been calculated at different pressure ratios, to investigate the loss generation (Figure 14). At ratios above the reference value of 0.568 , the reference profile has lower pressure losses in comparison to case C and D. Up to a 
pressure ratio of 0.55 both profiles (C and D) have a comparable value of losses. But the pressure losses of both cases are above the reference geometry. At lower ratios, especially below 0.5 , the optimized profiles are always showing lower pressure losses in comparison to the reference case. Especially, the case D is showing the best performance at ratios between 0.55 and 0.4 . It is interesting to observe, that the properties which are applied at specific boundary conditions are also valid at lower ratios.

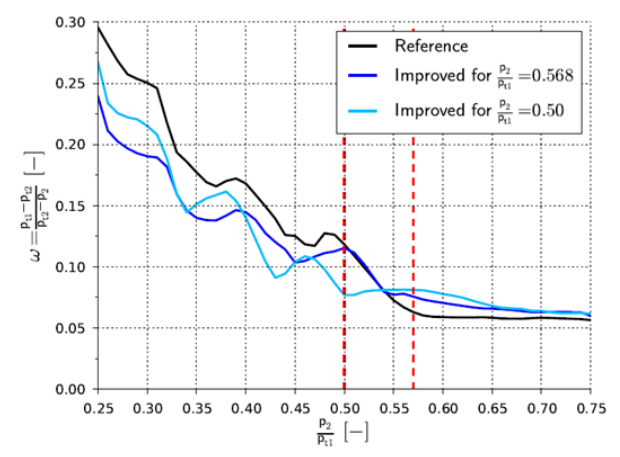

\section{Figure 14: Comparison of total pressure losses at different pressure ratios}

\section{CONCLUSIONS}

It can be stated, that the approach of prescribing the properties of the pressure distribution, which has been utilized for subsonic flow conditions, is also valid for transonic flow conditions. Furthermore, an application for the automated generation of efficient choked turbine cascades has been realized by prescribing the flow properties of intentional choked airfoils. The formation of a shock wave can be prescribed at the throat position for pre-defined boundary conditions.

The approach provides good results in $2 \mathrm{D}$, which also have been confirmed by $3 \mathrm{D}$ calculations. Especially the $3 \mathrm{D}$ calculations are showing that clear choke conditions over the whole span can be achieved by the approach, while the overall losses of the stage are not significantly increased. Overall, the comparison of the stage parameters has shown a good agreement to the reference case. Furthermore, the approach has been verified under lower static to total pressure ratios, which confirms the robustness of the approach in 2D.

Further investigations in 3D simulations will be necessary, as an individual design of airfoil sections at different span location may improve the choke conditions in radial direction.

The objective function $\mathrm{F}$ is suitable to prescribe the characteristics of transonic flow behavior, but do not consider any loss parameter. In this study a simple weighting between the efficiency and the function $F$ has been implemented to improve the performance of the profile. It is clear to see, that the value of the diffusion factor DFAC has a direct relation to the total losses in the blade passage. Furthermore, lower pressures on the suction side close to the trailing edge and weak, local shocks after the throat section are resulting in lower total loss generation. Further investigations will be necessary to implement a loss correlation into the function $\mathrm{F}$.

Also the behavior after the throat section will need further investigations, as a re-acceleration at this region seems to be beneficial for loss reduction and may avoid the formation of a shock wave side at the trailing edge (suction side).

\section{NOMENCLATURE}

2D two dimensional

3D three dimensional

B2B blade-to-blade

BC Boundary Conditions

CAD computer aided design

CFD computational fluid dynamics

DFAC diffusion factor

M Mach number

NGV nozzle guide vane

$\mathrm{P} \quad$ Power

TEPS trailing edge, suction side shock

TESS trailing edge, pressure side shock

i incidence

$\mathrm{r} \quad$ radius

SHERPA Simultaneous Hybrid Exploration that is robust, progressive and Adaptive

\section{GREEK SYMBOLS}

$\eta_{\mathrm{s}} \quad$ isentropic efficiency $\left(\eta_{\mathrm{s}}=\Delta h /\left(\Delta h+\Delta h_{V}\right)\right)$

$\eta \quad$ weighted pressure mixed efficiency

$\rho \quad$ degree of reaction $\left(\rho_{\mathrm{h}}=\Delta h_{R} / \Delta h\right)$

$\varphi \quad$ flow coefficient $\left(\varphi=c_{a x} / u_{2}\right)$

$\Psi \quad$ stage enthalpy coefficient $\left(\Psi_{\mathrm{h}}=2 \Delta h / u_{2}^{2}\right)$

$\Theta \quad$ total to total temperature ratio $\left(\mathrm{T}_{\mathrm{t} 2} / \mathrm{T}_{\mathrm{t} 0}\right)$

$\Pi \quad$ total to total pressure ratio $\left(\mathrm{p}_{\mathrm{t} 2} / \mathrm{p}_{\mathrm{t} 0}\right)$

\section{SUBSCRIPTS}

0, 1, 2 Stator Inlet, Stator Outlet, Rotor Outlet

$\mathrm{t} \quad$ total state

\section{ACKNOWLEDGMENTS}

Simulations in this paper have been carried out with STAR-CCM+ of Siemens PLM. Their support is gratefully acknowledged.

\section{REFERENCES}

[1] Baskharone, E., Principles of Turbomachinery in AirBreathing Engines. Cambridge: Cambridge University Press, 2006

[2] Bellucci, J., Rubechini, F., Arnone, A., Arcangeli, L., Maceli, N., Dossena, V., Optimization of a high-pressure steam turbine stage for a wide flow coefficient range. Proceedings of ASME Turbo Expo 2012, Copenhagen, Denmark

[3] Bohn, D., Heuer, T., Kusterer, K., Lang, G., Application of a Conjugate Fuid Flow and Heat Transfer Method in Thermal Design Process of a Convection Cooled Turbine Nozzel Vane. AMSE ASIA 97 Congress and Exhibition, Singapore, Singapore 
[4] Bohn, D., Ren, J., Teummers, C., Sell, M., Unsteady 3D-Numerical Investigation of the Influence of the Blading Design Process on the Stator-Rotor Interaction in a 2-Stage Turbine. Proceedings of ASME Turbo Expo 2005, RenoTahoe, Nevada, USA

[5] Braining, E., Weidtmann, K., Buehler, P., Braun, R., Lautenschlaeger, A., Improvement of an Automated Design Process and Investigation of the Optimization Approach and the Dependencies of the Individual Objectives. Proceedings of GPPS 2018, Montreal, Canada

[6] Cofer, J.I., Reinker, J.K., Sumner, W.J.. Advances in Steam Path Technology. Journal of Engineering for Gas Turbines and Power, Vol. 118, pp. 337-352, April 1996

[7] Denton, J.D., Xu, L., The Trailing Ede Loss of Transonic Turbine Blades. ASME Gas Turbine and Aeroengine Congress and Exposition 1989, Toronto, Canada

[8] Denton, J.D., The 1993 IGTI Scholar Lecture: Loss Mechanisms in Turbomachines. Journal of Turbomachinery, Vol. 115-4, 1993

[9] Giles, M., Stator/Rotor Interaction in a Transonic Turbine. AIAA Journal of Propulsion and Power, Vol. 6, Nr. 5,1990

[10] Graham, C., Kost, F. Shock Noundnary Layer Interaction on High Turning Transonic Turbine Cascades. ASME International Gas Turbine Conference and Exhibit and Solar Energy Conference, San Diego, USA, 1979

[11] Goel, S., Turbine Airfoil Optimization Using Quasi3D Analysis Codes. International Journal of Aerospace Engineering, Vol. 2009, Article ID 531358, January 2009

[12] Korpela, S., Principles of Turbomachinery, New Jersey, USA, 2011

[13] Mee, D., Baines, N., Oldfield, M., Dickens, T., An Examination of the Contributions to Loss on a Transonic Turbine Blade in Cascade. ASME Gas Turbine and Aeroengine Congress and Exposition 1990. Brussels, Belgium, 1990

[14] Paniagua, G., Yasa, T., Loma, A. de I., Unsteady Strong Shock Interactions in Transonic Turbine: Experimental and Numerical Analysis.. AIAA Journal of Turbomachinery, Vol. 137, Nr. 1, 2015

[15] Roache, P., Celik, I., Ghia, U., Freitas, C., Coleman, H., Raad, P., Procedure for Estimation and Reporting of Uncertainty Due to Discretization in CFD Applications (Announcement). ASME Journal of Fluid Engineering, Vol. 130, Nr. 7, 2008

[16] Wei, N., Significance of Loss Models in Aerothermodynamic Simulation for Axial Turbines, Royal Institute of Technology Stockholm, Doctoral Thesis, 2000

[17] Weidtmann, K., Buehler, P., Braining, E., Haj Ayed, A., Lin, G., High Efficieny Steam Turbine Design based on automated Design Space Exploration and Optimization Techniques. European Conference on Turbomachinery Fluid dynamics \& Thermodynamics 2017, Stockholm, Sweden [18] Weiß, A.P., Fottner, L., The influence of load distribution on secondary flow in straight turbine cascades. International Gas Turbine and Aeroengine Congress and Exposition 1993, Cincinnati, USA 